\title{
Matrix representations of Fibonacci-like sequences
}

Yasemin Tasyurdu

Department of Mathematics, Faculty of Science and Art, University of Erzincan Binali Yıldırım, Erzincan 24000, Turkey

Received: 01 October 2018, Accepted: 29 October 2018

Published online: 31 December 2018.

Abstract: In this paper, we define Fibonacci-Like matrix sequences $\left\{\widetilde{R}_{k, n}\right\}$ of Fibonacci-Like sequences associated with $k$-Pell, $k$-Pell Lucas and Modified $k$-Pell sequences and then we derive the matrices given $n$th general term of these matrix sequences. We also obtain Binet formulas, generating functions and some fundamental identities involving terms of these matrix sequences.

Keywords: k-Pell sequence, k-Pell Lucas sequence, Modified k-Pell sequences, Matrix.

\section{Introduction}

There are so many articles in the literature that concern the special number sequences such as Fibonacci, Lucas, Pell, Pell-Lucas, Modified Pell, Jacobsthal, Jacobsthal-Lucas satisfying second order linear recurrence relations and it has many important applications to diverse fields such as mathematic, computer science, physics, biology and statistics ([1], [4] [5], [9], [12], [13]). Also, it has been obtained a lot of studies on the new family of $k$-Fibonacci, $k$-Pell, $k$-Lucas, $k$-Jacobsthal numbers ([6], [7], [8], [14]).

The sequences $W_{n}(a, b ; p, q)$ are defined by the general recurrence relation

$$
W_{n}=p W_{n-1}-q W_{n-2}, \quad n \geq 2
$$

with $W_{0}=a, W_{1}=b$ and $a, b, p, q$, integers with $p \geq 1, q \neq 0$ by Horadam ([6], [7], [8]). In fact, in the Horadam notation, it can be written $k$-Pell sequences $\left\{P_{k, n}\right\}, k$-Pell-Lucas sequences $\left\{Q_{k, n}\right\}$ and Modified $k$-Pell sequences $\left\{q_{k, n}\right\}$ respectively by

$$
\begin{gathered}
P_{k, n}=W_{n}(0,1 ; 2,-k) \\
Q_{k, n}=W_{n}(2,2 ; 2,-k) \\
q_{k, n}=W_{n}(1,1 ; 2,-k) .
\end{gathered}
$$

The corresponding characteristic equation of equation (1) is

$$
x^{2}=p x-q
$$

and its roots are $r_{1}=\frac{p+\sqrt{p^{2}-4 q}}{2}$ and $r_{2}=\frac{p-\sqrt{p^{2}-4 q}}{2}$. For equations (2), (3) and (4), their roots are

$$
r_{1}=1+\sqrt{1+k} \text { and } r_{2}=1-\sqrt{1+k}
$$

and verify

$$
r_{1}+r_{2}=2, r_{1}-r_{2}=2 \sqrt{1+k} \text { and } r_{1} r_{2}=-k .
$$


Binet formulas of $k$-Pell sequences, $k$-Pell-Lucas sequences and Modified $k$-Pell sequences are given respectively by

$$
\begin{gathered}
P_{k, n}=\frac{r_{1}^{n}-r_{2}^{n}}{r_{1}-r_{2}} \\
Q_{k, n}=r_{1}^{n}+r_{2}^{n} \\
q_{k, n}=\frac{r_{1}^{n}+r_{2}^{n}}{2} .
\end{gathered}
$$

For any positive real number $k$, Fibonacci-Like sequences $\left\{R_{k, n}\right\}$ associated with $k$-Pell sequences, $k$-Pell-Lucas sequences and Modified $k$-Pell sequences are defined recurrently by

$$
R_{k, n}=2 R_{k, n-1}+k R_{k, n-2}, n \geq 2
$$

with $R_{k, 0}=2$ and $R_{k, 1}=1$ ([15]). Clearly $x^{2}-2 x-k=0$ is also the characteristic equation of $R_{k, n}=2 R_{k, n-1}+k R_{k, n-2}$ recurrence formula in equation (5) and $a$ and $b$ are its two roots where $a=1+\sqrt{1+k}$ and $b=1-\sqrt{1+k}$.

Many properties of these number sequences are deduced directly from elementary matrix algebra. The matrix method is used to get some properties for these number sequences. It has been considered new matrices which are based on Fibonacci and Pell sequences ([1], [2], [3], [10], [11]).

The main aim of the present article is to introduce matrix representations of Fibonacci-Like sequences which is similar to $k$-Pell, $k$-Pell-Lucas, Modified $k$-Pell sequences and known as Fibonacci-Like matrix sequences.

\section{Main Results}

\subsection{Fibonacci-Like Matrix Sequences $\left\{\widetilde{R}_{k, n}\right\}$}

In this section, we introduce definition of the Fibonacci-Like matrix sequences $\left\{\widetilde{R}_{k, n}\right\}$ by using Fibonacci-Like sequences associated with $k$-Pell, $k$-Pell-Lucas, Modified $k$-Pell sequences.

Throughout this paper, the symbol $\widetilde{R}_{k, n}$ is $n$th term of Fibonacci-Like matrix sequences associated with $k$-Pell, $k$-PellLucas, Modified $k$-Pell sequences and any positive real number $k$.

Definition 1.For any positive real number $k$, Fibonacci-Like matrix sequences $\left\{\widetilde{R}_{k, n}\right\}$ are defined recurrently by

$$
\widetilde{R}_{k, n}=2 \widetilde{R}_{k, n-1}+k \widetilde{R}_{k, n-2}, n \geq 2
$$

with initial conditions $\widetilde{R}_{k, 0}=\left(\begin{array}{cc}1 & 2 \\ 2 k & -3\end{array}\right), \widetilde{R}_{k, 1}=\left(\begin{array}{cc}2+2 k & 1 \\ k & 2 k\end{array}\right)$.

Example 1.A few terms of Fibonacci-Like matrix sequences $\left\{\widetilde{R}_{k, n}\right\}$ are

$$
\begin{aligned}
& \widetilde{R}_{k, 0}=\left(\begin{array}{cc}
1 & 2 \\
2 k & -3
\end{array}\right) \text {, } \\
& \widetilde{R}_{k, 1}=\left(\begin{array}{cc}
2+2 k & 1 \\
k & 2 k
\end{array}\right), \\
& \widetilde{R}_{k, 2}=\left(\begin{array}{cc}
4+5 k & 2+2 k \\
2 k+2 k^{2} & k
\end{array}\right), \\
& \widetilde{R}_{k, 3}=\left(\begin{array}{cc}
8+12 k+2 k^{2} & 4+5 k \\
4 k+5 k^{2} & 2 k+2 k^{2}
\end{array}\right)
\end{aligned}
$$

(C) 2018 BISKA Bilisim Technology 
Theorem 1. For $n \geq 0$ and any positive real number $k$, we have

$$
\widetilde{R}_{k, n}=\left(\begin{array}{cc}
R_{k, n+1} & R_{k, n} \\
k R_{k, n} & k R_{k, n-1}
\end{array}\right)
$$

where $R_{k, n}$ is nth term of Fibonacci-Like sequences.

Proof. We can use the induction method on $n$. Let us consider $R_{k,-1}=\frac{-3}{k}, R_{k, 0}=2, R_{k, 1}=1, R_{k, 2}=2+2 k$ from equation (5). For $n=0$ and $n=1$, we have

$$
\begin{aligned}
& \widetilde{R}_{k, 0}=\left(\begin{array}{cc}
R_{k, 1} & R_{k, 0} \\
k R_{k, 0} & k R_{k,-1}
\end{array}\right)=\left(\begin{array}{cc}
1 & 2 \\
2 k & -3
\end{array}\right) \\
& \widetilde{R}_{k, 1}=\left(\begin{array}{cc}
R_{k, 2} & R_{k, 1} \\
k R_{k, 1} & k R_{k, 0}
\end{array}\right)=\left(\begin{array}{cc}
2+2 k & 1 \\
k & 2 k
\end{array}\right) .
\end{aligned}
$$

So the proof is completed for $n=0$ and $n=1$. By iterating this procedure and considering induction steps, let us assume that the equation (7) holds for all $n \in \mathbb{Z}^{+}$. To finish the proof, we have to show that the equation (7) holds for $n+1$. By our assumption,

$$
\begin{aligned}
\widetilde{R}_{k, n+1} & =2 \widetilde{R}_{k, n}+k \widetilde{R}_{k, n-1} \\
& =2\left(\begin{array}{cc}
R_{k, n+1} & R_{k, n} \\
k R_{k, n} & k R_{k, n-1}
\end{array}\right)+k\left(\begin{array}{cc}
R_{k, n} & R_{k, n-1} \\
k R_{k, n-1} & k R_{k, n-2}
\end{array}\right) \\
& =\left(\begin{array}{cc}
2 R_{k, n+1}+k R_{k, n} & 2 R_{k, n}+k R_{k, n-1} \\
2 k R_{k, n}+k^{2} R_{k, n-1} & 2 k R_{k, n-1}+k^{2} R_{k, n-2}
\end{array}\right) \\
& =\left(\begin{array}{cc}
R_{k, n+2} & R_{k, n+1} \\
k R_{k, n+1} & k R_{k, n}
\end{array}\right)
\end{aligned}
$$

Hence, we obtain the desired result.

The $n$th general term of Fibonacci-Like matrix sequences $\left\{\widetilde{R}_{k, n}\right\}$ can be found by using the following theorem.

Theorem 2. For $n \geq 0$ and any positive real number $k$, the $n$th term of Fibonacci-Like matrix sequences $\left\{\widetilde{R}_{k, n}\right\}$ is given by

$$
\widetilde{R}_{k, n}=\left(\frac{\widetilde{R}_{k, 1}-\lambda_{2} \widetilde{R}_{k, 0}}{\lambda_{1}-\lambda_{2}}\right) \lambda_{1}^{n}-\left(\frac{\widetilde{R}_{k, 1}-\lambda_{1} \widetilde{R}_{k, 0}}{\lambda_{1}-\lambda_{2}}\right) \lambda_{2}^{n} .
$$

Proof. The characteristic equation of $\widetilde{R}_{k, n}=2 \widetilde{R}_{k, n-1}+k \widetilde{R}_{k, n-2}$ recurrence formula is $\lambda^{2}-2 \lambda-k=0$. The solutions of this equation are $\lambda_{1}$ and $\lambda_{2}$. The general term of Fibonacci-Like matrix sequences $\left\{\widetilde{R}_{k, n}\right\}$ may be expressed in the form

$$
\widetilde{R}_{k, n}=A \lambda_{1}^{n}+B \lambda_{2}^{n}
$$

for some coefficients $A$ and $B$. Giving to $n$ the values $n=0, n=1$ and solving this system of linear equations, it is obtained

$$
A=\left(\frac{\widetilde{R}_{k, 1}-\lambda_{2} \widetilde{R}_{k, 0}}{\lambda_{1}-\lambda_{2}}\right) \text { and } B=-\left(\frac{\widetilde{R}_{k, 1}-\lambda_{1} \widetilde{R}_{k, 0}}{\lambda_{1}-\lambda_{2}}\right)
$$

Using $A$ and $B$ in equation (8), we obtain

$$
\widetilde{R}_{k, n}=\left(\frac{\widetilde{R}_{k, 1}-\lambda_{2} \widetilde{R}_{k, 0}}{\lambda_{1}-\lambda_{2}}\right) \lambda_{1}^{n}-\left(\frac{\widetilde{R}_{k, 1}-\lambda_{1} \widetilde{R}_{k, 0}}{\lambda_{1}-\lambda_{2}}\right) \lambda_{2}^{n}
$$


which is as desired. Also, the roots $\lambda_{1}$ and $\lambda_{2}$ verifies the relation such as

$$
\begin{aligned}
\lambda_{1} \lambda_{2} & =-k \\
\lambda_{1}+\lambda_{2} & =2
\end{aligned}
$$

where $\lambda_{1}=1+\sqrt{1+k}$ and $\lambda_{2}=1-\sqrt{1+k}$.

\subsection{Generating functions for Fibonacci-like matrix sequences $\left\{\widetilde{R}_{k, n}\right\}$}

In this section, we give generating functions for Fibonacci-Like matrix sequences $\left\{\widetilde{R}_{k, n}\right\}$. A generating function $g(x)$ is a formal power series

$$
g(x)=\sum_{i=0}^{\infty} a_{n} x^{n}
$$

whose coefficients give the sequence $\left\{a_{0}, a_{1}, a_{2}, \ldots\right\}$. Given a generating function is the analytic expression for the $n t h$ term in the corresponding series. We will show that Fibonacci-Like matrix sequences $\left\{\widetilde{R}_{k, n}\right\}$ can be considered as the coefficients of the power series of the corresponding generating function.

Let us suppose that the terms of Fibonacci-Like matrix sequences $\left\{\widetilde{R}_{k, n}\right\}$ are coefficient of a potential series countered at the origin and consider the corresponding analytic functions $r_{k}(x)$ such that

$$
r_{k}(x)=\widetilde{R}_{k, 0}+\widetilde{R}_{k, 1} x+\widetilde{R}_{k, 2} x^{2}+\ldots+\widetilde{R}_{k, n} x^{n}+\ldots
$$

Then we can write

$$
\begin{gathered}
2 r_{k}(x) x=2 \widetilde{R}_{k, 0} x+2 \widetilde{R}_{k, 1} x^{2}+2 \widetilde{R}_{k, 2} x^{3}+\ldots+2 \widetilde{R}_{k, n} x^{n+1}+\ldots \\
k r_{k}(x) x^{2}=k \widetilde{R}_{k, 0} x^{2}+k \widetilde{R}_{k, 1} x^{3}+k \widetilde{R}_{k, 2} x^{4}+\ldots+k \widetilde{R}_{k, n} x^{n+2}+\ldots
\end{gathered}
$$

From the equations (9), (10) and (11), we obtain

$$
r_{k}(x)-2 r_{k}(x) x-k r_{k}(x) x^{2}=\widetilde{R}_{k, 0}+\widetilde{R}_{k, 1} x-2 \widetilde{R}_{k, 0} x
$$

where $\widetilde{R}_{k, i}=2 \widetilde{R}_{k, i-1}+k \widetilde{R}_{k, i-2}$ with initial $\widetilde{R}_{k, 0}=\left(\begin{array}{cc}1 & 2 \\ 2 k & -3\end{array}\right), \widetilde{R}_{k, 1}=\left(\begin{array}{cc}2+2 k & 1 \\ k & 2 k\end{array}\right)$ from Definition 1 . So the generating functions of Fibonacci-Like matrix sequences $\left\{\widetilde{R}_{k, n}\right\}$ are

$$
r_{k}(x)=\frac{\widetilde{R}_{k, 0}+\left(\widetilde{R}_{k, 1}-2 \widetilde{R}_{k, 0}\right) x}{1-2 x-k x^{2}}
$$

which is the desired.

\subsection{Some identities of Fibonacci-like matrix sequences $\left\{\widetilde{R}_{k, n}\right\}$}

In this section, we present some of the interesting properties of Fibonacci-Like matrix sequences $\left\{\widetilde{R}_{k, n}\right\}$ like Catalan's identity, Cassini's identity, d'Ocagne's identity.

Theorem 3. For any positive real number $k$,

$$
\sum_{i=0}^{n} \frac{\widetilde{R}_{k, i}}{\lambda^{i}}=\frac{1}{\lambda^{2}-2 \lambda-k}\left(\lambda \widetilde{R}_{k, 1}+\left(\lambda^{2}-2 \lambda\right) \widetilde{R}_{k, 0}\right)-\frac{1}{\lambda^{n}\left(\lambda^{2}-2 \lambda-k\right)}\left(\lambda \widetilde{R}_{k, n+1}+k \widetilde{R}_{k, n}\right)
$$


Proof. We can use Theorem 2 to prove. From Theorem 2, we obtain

$$
\sum_{i=0}^{n} \frac{\widetilde{R}_{k, i}}{\lambda^{i}}=\left(\frac{\widetilde{R}_{k, 1}-\lambda_{2} \widetilde{R}_{k, 0}}{\lambda_{1}-\lambda_{2}}\right) \sum_{i=0}^{n}\left(\frac{\lambda_{1}}{\lambda}\right)^{i}-\left(\frac{\widetilde{R}_{k, 1}-\lambda_{1} \widetilde{R}_{k, 0}}{\lambda_{1}-\lambda_{2}}\right) \sum_{i=0}^{n}\left(\frac{\lambda_{2}}{\lambda}\right)^{i}
$$

By using the definition of a geometric sequence, $\lambda_{1}+\lambda_{2}=2$ and $\lambda_{1} \lambda_{2}=-k$, we have

$$
\begin{aligned}
\sum_{i=0}^{n} \frac{\widetilde{R}_{k, i}}{\lambda^{i}} & =\left(\frac{\widetilde{R}_{k, 1}-\lambda_{2} \widetilde{R}_{k, 0}}{\lambda_{1}-\lambda_{2}}\right)\left(\frac{\lambda^{n+1}-\lambda_{1}^{n+1}}{\lambda^{n+1}\left(\frac{\lambda-\lambda_{1}}{\lambda}\right)}\right)-\left(\frac{\widetilde{R}_{k, 1}-\lambda_{1} \widetilde{R}_{k, 0}}{\lambda_{1}-\lambda_{2}}\right)\left(\frac{\lambda^{n+1}-\lambda_{2}^{n+1}}{\lambda^{n+1}\left(\frac{\lambda-\lambda_{2}}{\lambda}\right)}\right) \\
& =\left(\frac{\widetilde{R}_{k, 1}-\lambda_{2} \widetilde{R}_{k, 0}}{\lambda_{1}-\lambda_{2}}\right)\left(\lambda^{n+1}-\lambda_{1}^{n+1}\right)\left(\frac{1}{\lambda^{n}\left(\lambda-\lambda_{1}\right)}\right) \\
& -\left(\frac{\widetilde{R}_{k, 1}-\lambda_{1} \widetilde{R}_{k, 0}}{\lambda_{1}-\lambda_{2}}\right)\left(\lambda^{n+1}-\lambda_{2}^{n+1}\right)\left(\frac{1}{\lambda^{n}\left(\lambda-\lambda_{2}\right)}\right) \\
& =\left(\frac{\widetilde{R}_{k, 1}-\lambda_{2} \widetilde{R}_{k, 0}}{\lambda_{1}-\lambda_{2}}\right)\left(\lambda^{n+1}-\lambda_{1}^{n+1}\right)\left(\frac{\lambda-\lambda_{2}}{\lambda^{n}\left(\lambda-\lambda_{1}\right)\left(\lambda-\lambda_{2}\right)}\right) \\
& -\left(\frac{\widetilde{R}_{k, 1}-\lambda_{1} \widetilde{R}_{k, 0}}{\lambda_{1}-\lambda_{2}}\right)\left(\lambda^{n+1}-\lambda_{2}^{n+1}\right)\left(\frac{\lambda-\lambda_{1}}{\lambda^{n}\left(\lambda-\lambda_{2}\right)\left(\lambda-\lambda_{1}\right)}\right) \\
& =\frac{1}{\lambda^{n}\left(\lambda^{2}-2 \lambda-k\right)}\left(\frac{\widetilde{R}_{k, 1}-\lambda_{2} \widetilde{R}_{k, 0}}{\lambda_{1}-\lambda_{2}}\right)\left(\lambda^{n+1}-\lambda_{1}^{n+1}\right)\left(\lambda-\lambda_{2}\right) \\
& -\frac{1}{\lambda^{n}\left(\lambda^{2}-2 \lambda-k\right)}\left(\frac{\widetilde{R}_{k, 1}-\lambda_{1} \widetilde{R}_{k, 0}}{\lambda_{1}-\lambda_{2}}\right)\left(\lambda^{n+1}-\lambda_{2}^{n+1}\right)\left(\lambda-\lambda_{1}\right)
\end{aligned}
$$

If we rearrange the last equation, then we get

$$
\begin{aligned}
\sum_{i=0}^{n} \frac{\widetilde{R}_{k, i}}{\lambda^{i}} & =\frac{1}{\lambda^{n}\left(\lambda^{2}-2 \lambda-k\right)}\left[\left(\frac{\widetilde{R}_{k, 1}-\lambda_{2} \widetilde{R}_{k, 0}}{\lambda_{1}-\lambda_{2}}\right) \lambda^{n+1}\left(\lambda-\lambda_{2}\right)-\left(\frac{\widetilde{R}_{k, 1}-\lambda_{1} \widetilde{R}_{k, 0}}{\lambda_{1}-\lambda_{2}}\right) \lambda^{n+1}\left(\lambda-\lambda_{1}\right)\right] \\
& -\frac{1}{\lambda^{n}\left(\lambda^{2}-2 \lambda-k\right)}\left[\left(\frac{\widetilde{R}_{k, 1}-\lambda_{2} \widetilde{R}_{k, 0}}{\lambda_{1}-\lambda_{2}}\right) \lambda_{1}^{n+1} \lambda-\left(\frac{\widetilde{R}_{k, 1}-\lambda_{1} \widetilde{R}_{k, 0}}{\lambda_{1}-\lambda_{2}}\right) \lambda_{2}^{n+1} \lambda\right] \\
& +\frac{1}{\lambda^{n}\left(\lambda^{2}-2 \lambda-k\right)}\left[\left(\frac{\widetilde{R}_{k, 1}-\lambda_{2} \widetilde{R}_{k, 0}}{\lambda_{1}-\lambda_{2}}\right) \lambda_{1}^{n+1} \lambda_{2}-\left(\frac{\widetilde{R}_{k, 1}-\lambda_{1} \widetilde{R}_{k, 0}}{\lambda_{1}-\lambda_{2}}\right) \lambda_{2}^{n+1} \lambda_{1}\right] \\
& =\frac{1}{\left(\lambda^{2}-2 \lambda-k\right)} \frac{\lambda\left(\lambda_{1}-\lambda_{2}\right) \widetilde{R}_{k, 1}+\left(\lambda_{1}-\lambda_{2}\right)\left(\lambda^{2}-\lambda\left(\lambda_{1}+\lambda_{2}\right)\right) \widetilde{R}_{k, 0}}{\lambda_{1}-\lambda_{2}} \\
& -\frac{1}{\lambda^{n}\left(\lambda^{2}-2 \lambda-k\right)}\left(\lambda \widetilde{R}_{k, n+1}-\lambda_{1} \lambda_{2} \widetilde{R}_{k, n}\right) .
\end{aligned}
$$

and so we obtain

$$
\sum_{i=0}^{n} \frac{\widetilde{R}_{k, i}}{\lambda^{i}}=\frac{1}{\left(\lambda^{2}-2 \lambda-k\right)}\left(\lambda \widetilde{R}_{k, 1}+\left(\lambda^{2}-2 \lambda\right) \widetilde{R}_{k, 0}\right)-\frac{1}{\lambda^{n}\left(\lambda^{2}-2 \lambda-k\right)}\left(\lambda \widetilde{R}_{k, n+1}+k \widetilde{R}_{k, n}\right)
$$

which is the desired. 
Theorem 4. For any positive real number $k$ and $j>m$, we get

$$
\sum_{i=0}^{n} \widetilde{R}_{k, m i+j}=\frac{\widetilde{R}_{k, m n+m+j}-(-k)^{m}\left(\widetilde{R}_{k, m n+j}\right)+(-k)^{m}\left(\widetilde{R}_{k, j-m}\right)-\widetilde{R}_{k, j}}{\lambda_{1}^{m}+\lambda_{2}^{m}-(-k)^{m}-1}
$$

Proof. Let us take $U=\widetilde{R}_{k, 1}-\lambda_{2} \widetilde{R}_{k, 0}$ and $V=\widetilde{R}_{k, 1}-\lambda_{1} \widetilde{R}_{k, 0}$. Then we get

$$
\begin{aligned}
& \sum_{i=0}^{n} \widetilde{R}_{k, m i+j}=\sum_{i=0}^{n} \frac{U \lambda_{1}^{m i+j}-V \lambda_{2}^{m i+j}}{\lambda_{1}-\lambda_{2}} \\
& =\frac{1}{\lambda_{1}-\lambda_{2}}\left(U \lambda_{1}^{j} \sum_{i=0}^{n} \lambda_{1}^{m i}-V \lambda_{2}^{j} \sum_{i=0}^{n} \lambda_{2}^{m i}\right) \\
& =\frac{1}{\lambda_{1}-\lambda_{2}}\left(U \lambda_{1}^{j}\left(\frac{1-\lambda_{1}^{m(n+1)}}{1-\lambda_{1}^{m}}\right)-V \lambda_{2}^{j}\left(\frac{1-\lambda_{2}^{m(n+1)}}{1-\lambda_{2}^{m}}\right)\right) \\
& =\frac{\left(\frac{U \lambda_{1}^{m n+m+j}-V \lambda_{2}^{m n+m+j}}{\lambda_{1}-\lambda_{2}}\right)-\left(\frac{U \lambda_{1}^{m n+j}-V \lambda_{2}^{m n+j}}{\lambda_{1}-\lambda_{2}}\right)\left(\lambda_{1} \lambda_{2}\right)^{m}+\left(\frac{U \lambda_{1}^{j-m}-V \lambda_{2}^{j-m}}{\lambda_{1}-\lambda_{2}}\right)\left(\lambda_{1} \lambda_{2}\right)^{m}-\left(\frac{U \lambda_{1}^{j}-V \lambda_{2}^{j}}{\lambda_{1}-\lambda_{2}}\right)}{\lambda_{1}^{m}+\lambda_{2}^{m}-\left(\lambda_{1} \lambda_{2}\right)^{m}-1}
\end{aligned}
$$

If we use Theorem $2, \lambda_{1}+\lambda_{2}=2$ and $\lambda_{1} \lambda_{2}=-k$, we obtain

$$
\sum_{i=0}^{n} \widetilde{R}_{k, m i+j}=\frac{\widetilde{R}_{k, m n+m+j}-(-k)^{m}\left(\widetilde{R}_{k, m n+j}\right)+(-k)^{m}\left(\widetilde{R}_{k, j-m}\right)-\widetilde{R}_{k, j}}{\lambda_{1}^{m}+\lambda_{2}^{m}-(-k)^{m}-1}
$$

which is as desired.

\section{Theorem 5.}

$$
\widetilde{R}_{k, n} \widetilde{R}_{k, m}=\widetilde{R}_{k, m} \widetilde{R}_{k, n}
$$

Proof. We can use Theorem 1 to prove $\widetilde{R}_{k, n} \widetilde{R}_{k, m}=\widetilde{R}_{k, m} \widetilde{R}_{k, n}$. From Theorem 1 , we get

$$
\begin{aligned}
& \widetilde{R}_{k, n} \widetilde{R}_{k, m}=\left(\begin{array}{cc}
R_{k, n+1} & R_{k, n} \\
k R_{k, n} & k R_{k, n-1}
\end{array}\right)\left(\begin{array}{cc}
R_{k, m+1} & R_{k, m} \\
k R_{k, m} & k R_{k, m-1}
\end{array}\right) \\
& =\left(\begin{array}{cc}
R_{k, n+1} R_{k, m+1}+k R_{k, n} R_{k, m} & R_{k, n+1} R_{k, m}+k R_{k, n} R_{k, m-1} \\
k R_{k, n} R_{k, m+1}+k^{2} R_{k, n-1} R_{k, m} & k R_{k, n} R_{k, m}+k^{2} R_{k, n-1} R_{k, m-1}
\end{array}\right) \\
& =\left(\begin{array}{cc}
R_{k, n+1} R_{k, m+1}+k R_{k, n} R_{k, m} & \left(2 R_{k, n}+k R_{k, n-1}\right) R_{k, m}+k R_{k, n} R_{k, m-1} \\
k R_{k, n}\left(2 R_{k, m}+k R_{k, m-1}\right)+k^{2} R_{k, n-1} R_{k, m} & k R_{k, n} R_{k, m}+k^{2} R_{k, n-1} R_{k, m-1}
\end{array}\right) \\
& =\left(\begin{array}{cc}
R_{k, m+1} R_{k, n+1}+k R_{k, m} R_{k, n} & \left(2 R_{k, m}+k R_{k, m-1}\right) R_{k, n}+k R_{k, m} R_{k, n-1} \\
k R_{k, m}\left(2 R_{k, n}+k R_{k, n-1}\right)+k^{2} R_{k, n} R_{k, m-1} & k R_{k, n} R_{k, m}+k^{2} R_{k, n-1} R_{k, m-1}
\end{array}\right) \\
& =\left(\begin{array}{cc}
R_{k, m+1} R_{k, n+1}+k R_{k, m} R_{k, n} & R_{k, m+1} R_{k, n}+k R_{k, m} R_{k, n-1} \\
k R_{k, m} R_{k, n+1}+k^{2} R_{k, m-1} R_{k, n} & k R_{k, m} R_{k, n}+k^{2} R_{k, m-1} R_{k, n-1}
\end{array}\right) \\
& =\left(\begin{array}{cc}
R_{k, m+1} & R_{k, m} \\
k R_{k, m} & k R_{k, m-1}
\end{array}\right)\left(\begin{array}{cc}
R_{k, n+1} & R_{k, n} \\
k R_{k, n} & k R_{k, n-1}
\end{array}\right) \\
& =\widetilde{R}_{k, m} \widetilde{R}_{k, n} \text {. }
\end{aligned}
$$

So the proof is completed.

As the other way, Theorem 2 can be used for proof of Theorem 5 too.

Theorem 6. (Catalan's Identity) For $0 \leq r \leq n$,

$$
\widetilde{R}_{k, n-r} \widetilde{R}_{k, n+r}=\widetilde{R}_{k, n}^{2}
$$


where any positive real number $k$.

Proof. Let us take $U=\widetilde{R}_{k, 1}-\lambda_{2} \widetilde{R}_{k, 0}$ and $V=\widetilde{R}_{k, 1}-\lambda_{1} \widetilde{R}_{k, 0}$. By using Theorem 2, we can write

$$
\widetilde{R}_{k, n-r} \widetilde{R}_{k, n+r}-\widetilde{R}_{k, n}^{2}=\left(\frac{U \lambda_{1}^{n-r}-V \lambda_{2}^{n-r}}{\lambda_{1}-\lambda_{2}}\right)\left(\frac{U \lambda_{1}^{n+r}-V \lambda_{2}^{n+r}}{\lambda_{1}-\lambda_{2}}\right)-\left(\frac{U \lambda_{1}^{n}-V \lambda_{2}^{n}}{\lambda_{1}-\lambda_{2}}\right)^{2} .
$$

If we arrange the last equation, we obtain

$$
\widetilde{R}_{k, n-r} \widetilde{R}_{k, n+r}-\widetilde{R}_{k, n}=\frac{U V\left(2\left(\lambda_{1} \lambda_{2}\right)^{n}-\lambda_{1}^{n-r} \lambda_{2}^{n+r}-\lambda_{1}^{n+r} \lambda_{2}^{n-r}\right)}{\left(\lambda_{1}-\lambda_{2}\right)^{2}}
$$

On the other hand, we get

$$
U V=\left(\widetilde{R}_{k, 1}-\lambda_{2} \widetilde{R}_{k, 0}\right)\left(\widetilde{R}_{k, 1}-\lambda_{1} \widetilde{R}_{k, 0}\right)=[0]_{2 x 2}
$$

by using $\widetilde{R}_{k, 0}=\left(\begin{array}{cc}1 & 2 \\ 2 k & -3\end{array}\right)$ and $\widetilde{R}_{k, 1}=\left(\begin{array}{cc}2+2 k & 1 \\ k & 2 k\end{array}\right)$ from Definition 1. Hence, we obtain

$$
\widetilde{R}_{k, n-r} \widetilde{R}_{k, n+r}=\widetilde{R}_{k, n}^{2}
$$

which is the required result.

Note that for $r=1$ in obtained Catalan's identity, we have the Cassini's identity for Fibonacci-Like matrix sequences $\left\{\widetilde{R}_{k, n}\right\}$. So we can write following corollary.

Corollary 1. (Cassini's Identity)

$$
\widetilde{R}_{k, n-1} \widetilde{R}_{k, n+1}=\widetilde{R}_{k, n}^{2} .
$$

Theorem 7. For any real number $k$ and $n<m, s \leq t$,

$$
\widetilde{R}_{k, m+t} \widetilde{R}_{k, n+s}=\widetilde{R}_{k, m+s} \widetilde{R}_{k, n+t} .
$$

Proof. Let us take $U=\widetilde{R}_{k, 1}-\lambda_{2} \widetilde{R}_{k, 0}$ and $V=\widetilde{R}_{k, 1}-\lambda_{1} \widetilde{R}_{k, 0}$. By using Theorem 2, we obtain

$$
\begin{aligned}
\widetilde{R}_{k, m+t} \widetilde{R}_{k, n+s}-\widetilde{R}_{k, m+s} \widetilde{R}_{k, n+t} & =\left(\frac{U \lambda_{1}^{m+t}-V \lambda_{2}^{m+t}}{\lambda_{1}-\lambda_{2}}\right)\left(\frac{U \lambda_{1}^{n+s}-V \lambda_{2}^{n+s}}{\lambda_{1}-\lambda_{2}}\right) \\
& -\left(\frac{U \lambda_{1}^{m+s}-V \lambda_{2}^{m+s}}{\lambda_{1}-\lambda_{2}}\right)\left(\frac{U \lambda_{1}^{n+t}-V \lambda_{2}^{n+t}}{\lambda_{1}-\lambda_{2}}\right) \\
& =\frac{\left(U^{2} \lambda_{1}^{m+t+n+s}-U V \lambda_{1}^{m+t} \lambda_{2}^{n+s}-U V \lambda_{1}^{n+s} \lambda_{2}^{m+t}+V^{2} \lambda_{2}^{m+t+n+s}\right)}{\left(\lambda_{1}-\lambda_{2}\right)^{2}} \\
& +\frac{\left(-U^{2} \lambda_{1}^{m+t+n+s}+U V \lambda_{1}^{m+s} \lambda_{2}^{n+t}+U V \lambda_{1}^{n+t} \lambda_{2}^{m+s}-V^{2} \lambda_{2}^{m+t+n+s}\right)}{\left(\lambda_{1}-\lambda_{2}\right)^{2}} \\
& =\frac{U V\left(\lambda_{1}^{t} \lambda_{2}^{s}-\lambda_{1}^{s} \lambda_{2}^{t}\right)\left(\lambda_{1}^{n} \lambda_{2}^{m}-\lambda_{1}^{m} \lambda_{2}^{n}\right)}{\left(\lambda_{1}-\lambda_{2}\right)^{2}} .
\end{aligned}
$$

Consequently, from $U V=\left(\widetilde{R}_{k, 1}-\lambda_{2} \widetilde{R}_{k, 0}\right)\left(\widetilde{R}_{k, 1}-\lambda_{1} \widetilde{R}_{k, 0}\right)=[0]_{2 x 2}$, we get

$$
\widetilde{R}_{k, m+t} \widetilde{R}_{k, n+s}=\widetilde{R}_{k, m+s} \widetilde{R}_{k, n+t}
$$

as required.

Note that for $t=0$ and $s=1$ in Theorem 7, we obtain d'Ocagne's identity. So we can write following corollary. 
Corollary 2. (d'Ocagne's Identity) For $n<m$,

$$
\widetilde{R}_{k, m} \widetilde{R}_{k, n+1}-\widetilde{R}_{k, m+1} \widetilde{R}_{k, n}=[0]_{2 \times 2}
$$

\section{Acknowledgement}

The author declare that they have no competing interests.

The author express their sincere thanks to the referee for his/her careful reading and suggestions that helped to improve this paper.

\section{References}

[1] N. Bicknell, A primer on the Pell sequence and related sequence, Fibonacci Quarterly, 13(4), (1975), 345-349.

[2] A. Dasdemir, On the Pell, Pell-Lucas and Modified Pell Numbers by Matrix Method, Applied Mathematical Sciences, 5(64), (2011), 3173-3181.

[3] J. Ercolano, Matrix generators of Pell sequences, The Fibonacci Quarterly, 17(1), (1979),71-77.

[4] V. E. Hoggatt, Fibonacci and Lucas Numbers. A publication of the Fibonacci Association, Boston, Houghton Mi- in Company, (OCoLC) 654299031, (1969).

[5] A. F. Horadam, Applications of Modified Pell Numbers to Representations, Ulam Quart., 3, (1994), 34-53.

[6] A. F. Horadam, Basic properties of a certain generalized sequences of numbers, Fibonacci Quarterly, 3(3), (1965), 161-176.

[7] A. F. Horadam, Generating functions for powers of numbers, Duke Math. J. 32(3), (1965), 437-446.

[8] A. F. Horadam, Special properties of the sequence $w_{n}=(a, b ; p, q)$, Fibonacci Quarterly, 5(4), (1967), 424-434.

[9] A. F. Horadam, Jacobsthal Representation Numbers, The Fibonacci Quarterly, 34(1), (1996), 40-54.

[10] D. Kalman, Generalized Fibonacci numbers by matrix methods, The Fibonacci Quarterly, 20(1), (1982), 73-76.

[11] E. Kılıç and D. Taşçı, ”The Linear Algebra of The Pell Matrix”, Bol. Soc. Mat. Mexicana, 3(11); (2005).

[12] T. Koshy, "Fibonacci and Lucas Numbera with Applications", Wiley- Interscience Publications, 2001.

[13] N. N. Vorobiov, ”Números de Fibonacci”, Editora MIR, URSS, (1974).

[14] Y. Tasyurdu, N. Cobanoglu and Z. Dilmen, On The a New Family of k-Fibonacci Numbers, Erzincan University Journal of Science and Thechnology, 9(1), (2016), 95-101.

[15] A. A. Wani, S. A. Bhat and G.P.S. Rathore, Fibonacci-Like Sequences Associated With k-Pell, k-Pell-Lucas and Modified k-Pell Sequences, Journal of Applied Mathematics and Computational Mechanics, 16(2), (2017), 159-171. 